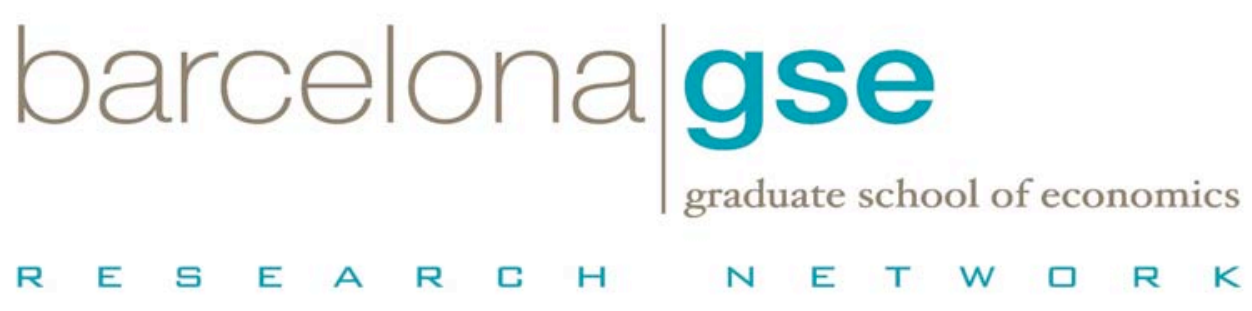

Single-dipped preferences

Salvador Barberà

Dolors Berga

Bernardo Moreno

December 31st, 2009

Barcelona Economics Working Paper Series

Working Paper $n^{\circ} 418$ 


\title{
Single-dipped preferences'
}

\author{
Salvador Barberà ${ }^{\dagger}$ \\ Dolors Berga \\ and \\ Bernardo Moreno ${ }^{\S}$
}

December 31st, 2009

\begin{abstract}
We characterize the set of all individual and group strategy-proof rules on the domain of all single-dipped preferences on a line. For rules defined on this domain, and on several of its subdomains, we explore the implications of these strategy-proofness requirements on the maximum size of the rules' range. We show that when all single-dipped preferences are admissible, the range must contain two alternatives at most. But this bound changes as we consider different subclasses of single-dipped preferences: we provide examples of subdomains admitting strategyproof rules with larger ranges. We establish exact bounds on the maximal size of strategy-proof functions on each of these domains, and prove that the relationship between the sizes of the subdomains and those of the ranges of strategy-proof functions on them need not be monotonic. Our results exhibit a sharp contrast between the structure of strategy-proof rules defined on subdomains of singledipped preferences and those defined on subsets of single-peaked ones.
\end{abstract}

JEL Classification Number: D71.

Keywords: Strategy-proof, Group strategy-proof, Binary range rules, Single-dipped.

\footnotetext{
${ }^{1}$ Salvador Barberà gratefully acknowledges support from the Spanish Ministry of Science and Innovation through grant "Consolidated Group-C" ECO2008-04756, and by the Generalitat de Catalunya, Departament d'Universitats, Recerca i Societat de la Informació through the Distinció per a la Promoció de la Recerca Universitària, grant SGR2009-0419 and the Barcelona GSE Research Network. Dolors Berga acknowledges the support of the Spanish Ministry of Science and Innovation through grant SEJ2007-60671 and of Generalitat de Catalunya, through grant SGR2009-0189. She also acknowledges the Research Recognition Programme of the Barcelona GSE. Bernardo Moreno acknowledges financial support from Junta de Andalucía through Proyecto de Excelencia SEJ1645 and the ECO2008-03674 from the Spanish Ministry of Science and Innovation.

${ }^{\dagger}$ MOVE, Universitat Autònoma de Barcelona, Barcelona GSE, Edifici B, 08193 Bellaterra, Spain. E-mail: salvador.barbera@uab.cat

${ }^{\ddagger}$ Departament d’Economia, Campus de Montilivi, Universitat de Girona, 17071 Girona, Spain. E-mail: dolors.berga@udg.edu.

$\S$ Departamento de Teoría e Historia Económica, Facultad de Ciencias Económicas y Empresariales, Campus de El Ejido, 29071 Málaga, Spain. E-mail: bernardo@uma.es
} 


\section{Introduction}

Single-dipped preferences naturally arise in the presence of a public bad. Consider, for example, the decision on where to locate a facility whose neighborhood is undesirable, like a prison, a dumping site or an incineration plant. It is natural to assume that the worse allocation for each agent is the one that places the facility right by their home, and that locations become better as they place it further away. ${ }^{1}$

When location can be identified with a point on a line, this gives rise to single-dipped preferences. We concentrate on this case, though we can also think of natural extensions to two-dimensional spaces, or even k-dimensional ones, under alternative interpretations. ${ }^{2}$

The purpose of this paper is to define and to partially characterize strategyproof rules on the domain of single-dipped preferences, and also on its subdomains.

It is natural to compare our setup and our results with those obtained in the case of single-peaked preferences, a much more studied but somewhat dual case that arises in cases when proximity to the public facility to be located is desirable, rather than a bad. In fact, these two restrictions also arise naturally from assumptions on the fundamentals of very simple models, other than the location example we just started with. For example, when agents with linear preferences must choose from the downward sloping frontier of a set of feasible alternatives in a two-good model, one can identify this frontier with a segment of the line, over which individual preferences will be single-dipped, or single-peaked, depending on whether the frontier is convex or concave.

\footnotetext{
${ }^{1}$ The appropriate specification of what is meant by "being further" may not only involve standard distance, but also other considerations, like the direction of prevailing winds, in the case of incineration plants, the existence of natural barriers in some directions and not in others, etc, $\ldots$

${ }^{2}$ Another interesting model giving rise to single-dipped preferences is provided by Manjunath (2009) and involves the division of a given budget for public goods among two sites.
} 


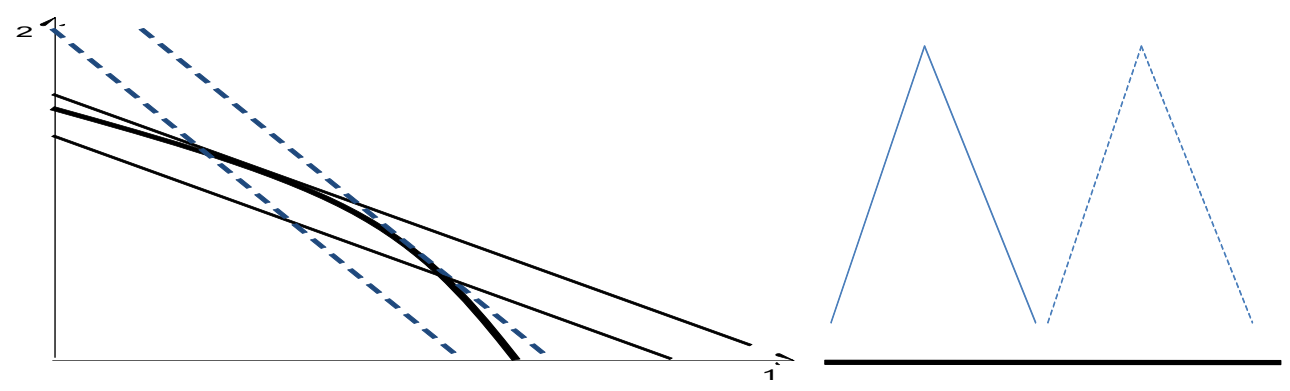

Figure 1: Single-peaked preferences over a concave frontier

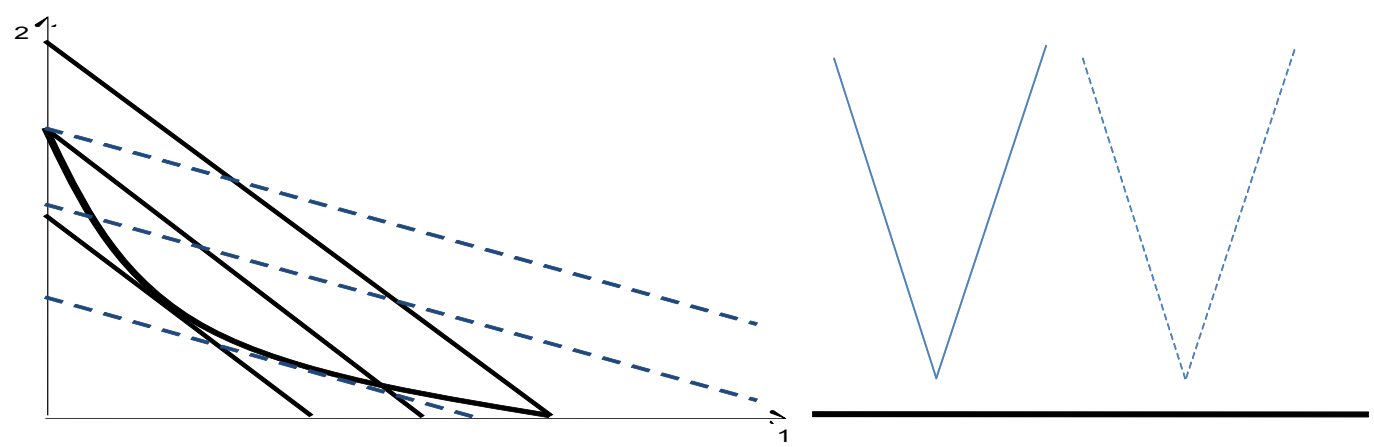

Figure 2: Single-dipped preferences over a convex frontier

The set of all strategy-proof rules whose domain includes all single-peaked preferences was characterized by Moulin (1980). These rules, called generalized median voter schemes, constitute a rich class and contain many alternative procedures.

One feature that is common to both domains is that all rules that are strategy-proof on them or on any of their subdomains are also group strategyproof. This is because both satisfy a condition called sequential inclusion (see Barberà, Berga, and Moreno, 2009a) that guarantees the equivalence of these two otherwise different incentive-compatibility requirements.

However, this coincidence regarding the equivalence of individual and group strategy-proofness does not carry over other characteristics of our rules on these two domains. In particular, they dramatically differ regarding the characteristics of their ranges. In the case of single-peaked preferences and 
their subdomains, the range of strategy-proof functions can often consist of the whole set of alternatives. ${ }^{3}$ By contrast, we will show that in domains where all single-dipped preferences are feasible, the range of strategy-proof rules contain at most two alternatives. This striking limitation is only one instance of a more general fact: that the size of the maximal ranges for strategy-proof rules on families of single-dipped preferences is endogenously predetermined by the nature of the domains that it must be defined on. This leads us to consider different subdomains of single-dipped preferences, to establish the maximal sizes that the range of strategy-proof rules over them, and to exhibit examples of rules where these sizes, that can certainly be larger than two, are effectively attained.

Of course, all strategy proof rules on the domain of all single-dipped preferences are also strategy-proof on its subdomains. But as these become more restrictive, new strategy-proof rules may arise. Some of those that we describe are of special interest, and all of them will still be group strategyproof, as a result of our already mentioned equivalence result.

The work of Peremans and Storcken (1999) is an important predecessor of ours. Indeed, they already pointed at the equivalence between individual and group strategy-proofness in subdomains of single-dipped preferences, a phenomenon that we can rationalize and extend because we now can check that the condition of sequential inclusion is satisfied on any such subset of profiles. Peremans and Storcken started a systematic study of restrictions imposed by strategy-proofness on the ranges of rules defined for special subdomains of single-dipped preferences. We improve on the bound that they propose and we analyze several new cases for which we can also provide tight results. However, there is no denial that theirs is a pioneer study of the subject.

A recent paper by Manjunath (2009) provides a result that is very similar to the one we obtain for the domain of all single-dipped preferences, in that it also shows that the range of rules must be of size two, and also provides a characterization of all strategy-proof rules in that case. The main differences are that, unlike Manjunath, we do not impose the requirement that rules are unanimous and we do not concentrate on a bounded interval in the real line, and this allows us to be slightly more general on those points. Another

\footnotetext{
${ }^{3}$ This is true for rules defined on the full domain of single-peaked preferences, and for many other subdomains. To get a full range it is sufficient (though not necessary) that any alternative is top for each agent at some admissible preference.
} 
difference is that we use a result of our own in the characterization of the rules (see Barberà, Berga, and Moreno, 2009b), while he appeals to a previous result by Larsson and Svensson (2006). In that specific aspect, our paper and Manjunath's seem to be nicely complementary. After that our contribution, as already explained, takes the direction of exploring new subdomains and to provide additional results on the maximal sizes of ranges allowed by the strategy-proofness requirement.

The paper is organized as follows. Section 2 contains the model and definitions while Section 3 encompasses the results concerning the set of all single-dipped preferences. Finally, in Section 4 we gather some examples of rules for subdomains of single-dipped preferences and also the results concerning the size of the range of strategy-proof rules on such subdomains.

\section{The setup and definitions}

Let $A$ be a finite set of alternatives ${ }^{4}$ and $N=\{1, \ldots, n\}$ be a finite set of agents. Let $\mathcal{R}$ denote the set of admissible preferences for any agent $i \in N$, such that individual preferences are preorders (complete, reflexive, and transitive binary relations on $A$ ). We denote by $R_{i} \in \mathcal{R}$ an admissible preference relation for agent $i$ and let as usual, $P_{i}$ and $I_{i}$ be the strict and the indifference part of $R_{i}$, respectively. A preference profile, denoted by $R_{N}=\left(R_{1}, \ldots, R_{n}\right)$, is an element of $\mathcal{R}^{n}=\mathcal{R} \times \ldots \times \mathcal{R}$. Let $C, S \subset N$ be two coalitions such that $C \subset S$ and $c$ and $s$ denote their cardinality. We will write the subprofile $R_{S}=\left(R_{C}, R_{S \backslash C}\right) \in \mathcal{R}^{s}$ when we want to stress the role of coalition $C$ in $S$. Then the subprofiles $R_{C} \in \mathcal{R}^{c}$ and $R_{S \backslash C} \in \mathcal{R}^{s-c}$ denote the preferences of agents in $C$ and in $S \backslash C$, respectively. In the case, where we denote full preference profile (that is, when $S=N$ ), we simplify notation by using $\left(R_{C}, R_{N \backslash C}\right)$ as $\left(R_{C}, R_{-C}\right)$.

We now define formally the notion of single-dipped preferences, of singledipped profiles and of single-dipped preference domains, relative to a given order of alternatives.

Definition 1 A preference relation of individual $i \in N, R_{i}$ is single-dipped on A relative to a linear order $>$ of the set of alternatives if

\footnotetext{
${ }^{4}$ All results in Section 3 hold if $A$ is any closed interval in the real line or the real line itself.
} 
(1) $R_{i}$ has a unique minimal element $d_{i}(A)$, called the dip of $i$, and

(2) For all $d_{i}(A)$ and for all $y, z \in A$

$$
\left[d_{i}(A) \geqslant y>z \text { or } z>y \geqslant d_{i}(A)\right] \rightarrow z P_{i} y .
$$

Definition 2 A preference profile is single-dipped relative to a linear order $>$ of alternatives if the preferences of all individuals satisfy the conditions in Definition 1, relative to the same order. Let $\mathcal{D}_{>}$denote the set of all single-dipped preferences profiles relative to $>$.

Definition 3 A preference domain is a subset of preference profiles. A (preference) domain is single-dipped if all the profiles it contains (and thus, all the preferences of all agents at all of its profiles) are single-dipped relative to the same linear order. In our notation, a domain is single-dipped if it takes the form $\mathcal{R}_{>}^{n}=\mathcal{R}_{>} \times \ldots \times \mathcal{R}_{>} \subseteq \mathcal{D}_{>}$.

Note that single-dipped preferences satisfy one of the forms of value restriction, as defined in Sen and Pattanaik (1969).

A social choice function (or a rule) is a function $f: \mathcal{R}_{>}^{n} \rightarrow A$. Let $A_{f}$ denote the range of the social choice function $f$.

We will focus on rules that are nonmanipulable, neither by a single agent nor by a coalition of agents. We first define what we mean by a manipulation and then we introduce the well known concepts of strategy-proofness and group strategy-proofness.

Definition $4 A$ social choice function $f$ is group manipulable on $\mathcal{R}_{>}^{n}$ at $R_{N} \in \mathcal{R}_{>}^{n}$ if there exists a coalition $C \subset N$ and $R_{C}^{\prime} \in \mathcal{R}_{>}^{c}\left(R_{i}^{\prime} \neq R_{i}\right.$ for any $i \in C)$ such that $f\left(R_{C}^{\prime}, R_{-C}\right) P_{i} f\left(R_{N}\right)$ for all $i \in C$. We say that $f$ is individually manipulable if there exists a possible manipulation where coalition $C$ is a singleton. ${ }^{5}$

Definition $5 A$ social choice function $f$ is group strategy-proof on $\mathcal{R}_{>}^{n}$ if $f$ is not group manipulable for any $R_{N} \in \mathcal{R}_{>}^{n}$. Similarly, $f$ is strategy-proof if it is not individually manipulable.

\footnotetext{
${ }^{5}$ Our definition requires that all agents in a coalition that manipulates should obtain a strictly positive benefit from doing so. We consider this requirement compelling, since it leaves no doubt regarding the incentives for each member of the coalition to participate in a collective deviation from truthful revelation. For the analysis of a stronger version of group strategy-proofness in the present setting, see Manjunath (2009).
} 
Notice that the domains of our social choice functions will always have the form of a Cartesian product. This is necessary to give meaning to our definitions of individual and group strategy-proofness. ${ }^{6}$

Barberà, Berga, and Moreno (2009a) showed that any subset of singledipped preferences profiles satisfies a domain condition called sequential inclusion. They also showed that for domains satisfying such condition, strategy-proofness and group strategy-proofness turn out to be equivalent (see next Remark 1). From now on, we will use strategy-proofness and group strategy-proofness indistinctly.

Remark 1 (See Theorem 1 in Barberà, Berga, and Moreno, 2009a) Any strategy-proof rule $f$ defined on $\mathcal{R}_{>}^{n} \subseteq \mathcal{D}_{>}$is group strategy-proof.

\section{Strategy-proofness on $\mathcal{D}_{>}$}

In this section we provide a characterization of all strategy-proof rules on the set of all single-dipped preferences. After reminding the reader of some facts that are relevant for our purposes, we establish that the range of these functions must contain at most two alternatives. That is, all non-constant strategy-proof rules on the domain should establish which pair of preselected alternatives prevails. We then combine this fact with a characterization result on strategy-proof rules with range two, in order to get the characterization.

We start by stating a well-known result that applies for any domain of preferences, not only for single-dipped ones. We include its straightforward proof, for the sake of completeness.

Definition 6 Let $\times_{i \in N} \mathcal{R}_{i} \subseteq \mathcal{R}^{n}$ such that $\mathcal{R}_{i}$ may differ from $\mathcal{R}_{j}$ for any $i, j \in N$. A social choice function $f$ is Pareto efficient on $A_{f}$ if for any $R_{N} \in \times_{i \in N} \mathcal{R}_{i} \subseteq \mathcal{R}^{n}$ there is no alternative $x \in A_{f}$ such that $x P_{i} f\left(R_{N}\right)$ for all $i \in N$.

\footnotetext{
${ }^{6}$ This is why we choose to define single-dipped domains in the way we do, that is, relative to the same order of alternatives for all profiles. An alternative definition would say that a domain is single-dipped if for each profile there exists an order of alternatives relative to which this profile is single-dipped. However, such definition would not guarantee the Cartesian structure of the domain.
} 
Lemma 1 Any group strategy-proof social choice function $f$ on $\times_{i \in N} \mathcal{R}_{i}$ is Pareto efficient on the range. ${ }^{7}$

Proof. By contradiction suppose there exist $R_{N} \in \times_{i \in N} \mathcal{R}_{i}$ and $x \in A_{f}$ such that $x P_{i} f\left(R_{N}\right)$ for all $i \in N$. Let $R_{N}^{\prime}$ such that $x=f\left(R_{N}^{\prime}\right)$. Let $S \subset N$ be the set of agents $i$ such that $R_{i} \neq R_{i}^{\prime}$. Note that $S \neq \varnothing$. Then, $S$ manipulates $f$ at $R_{N}$ via $R_{S}^{\prime}$ which contradicts group strategy-proofness.

Peremans and Storcken (1999) show that as a consequence of their Lemmas 3 and 4, any given strategy-proof rule on any subdomain of single-dipped preferences has at most $2^{n}$ alternatives in the range. In particular this upper bound is determined by the cardinality of the set of admissible profiles when each agent has only two admissible preferences (leftist, i.e., the order of alternatives according to the "smaller than" relation and rightist, i.e., the order of alternatives according to the "greater than" relation). This upper bound can be attained for this very restricted domain provided that there are enough feasible alternatives. We obtain other bounds for the size of the range, which depend on the size and nature of the preferences that constitute admissible domains. The first bound applies when all single-dipped preferences are admissible and it is expressed in Theorem 1: only two alternatives may be in the range! Other interesting bounds for the sizes of ranges under different subdomains of single-dipped preferences are obtained in Section 4. In the next Theorem 1 we refine such Peremans and Storcken (1999)'s result by obtaining a more accurate upper bound when all single-dipped preference profiles are admissible.

Theorem 1 Any strategy-proof social choice function $f$ on $\mathcal{D}_{>}$is such that $\# A_{f} \leq 2$.

Note, as we show in the following example, that the result in Theorem 1 can not be generalized to all subset of single-dipped preferences. In the next section we stress this point.

Example 1 Let $N=\{1,2\}, A=\{x, y, z\}$ where $z>y>x$ and $\mathcal{R}_{>}=$ $\left\{R, R^{\prime}, \bar{R}\right\}$ such that $x P y P z, x P^{\prime} z P^{\prime} y$, and $z \bar{P} y \bar{P} x$ as presented in the fol-

\footnotetext{
${ }^{7}$ The definition of group strategy-proofness is valid for any Cartesian product of individual domain of preferences.
} 
lowing table:

\begin{tabular}{|l|l|l|}
\hline$R$ & $R^{\prime}$ & $\bar{R}$ \\
\hline$x$ & $x$ & $z$ \\
\hline$y$ & $z$ & $y$ \\
\hline$z$ & $y$ & $x$ \\
\hline
\end{tabular}

Note that $\mathcal{R}_{>}^{2}$ is a subset of single-dipped profiles relative to the above defined order of alternatives. The social choice function $f$ on $\mathcal{R}_{>}^{2}$ defined below is strategy-proof and the size of its range $\left(\# A_{f}\right)$ is $3>2$.

\begin{tabular}{|l|l|l|l|}
\hline$f$ & $R_{2}$ & $R_{2}^{\prime}$ & $\bar{R}_{2}$ \\
\hline$R_{1}$ & $x$ & $x$ & $y$ \\
\hline$R_{1}^{\prime}$ & $x$ & $x$ & $z$ \\
\hline $\bar{R}_{1}$ & $y$ & $z$ & $z$ \\
\hline
\end{tabular}

Before proving Theorem 1, we need to introduce the following fact, some useful notation, and other interesting results.

Fact Note that for any triple of alternatives in $A$, say $t$, formed by $x, y, z$ where $z>y>x$, the restriction of any $R_{N} \in \mathcal{R}_{>}^{n} \subseteq \mathcal{D}_{>}$to $x, y, z$, say $R_{N, t}=\left(R_{1, t}, \ldots, R_{n, t}\right)$, reduces to one of the following kinds of admissible individual preference relations:

$$
\begin{aligned}
& x P^{1} y, x P^{1} z, \text { and } y R^{1} z \\
& x P^{2} z P^{2} y \\
& x I^{3} z, \text { and } z P^{3} y \\
& z P^{4} x P^{4} y \\
& z P^{5} y, z P^{5} x \text {, and } y R^{5} x .
\end{aligned}
$$

Informally, we call such individual preferences as type $l$ preferences, where $l \in\{1,2,3,4,5\}$. We will say that type 1 preferences in the list above are "leftist on $t$ " and that type 5 preferences are "rightist on $t$ ".

Notation: Take a triple $t$ formed by $x, y, z \in A$ and $S \subset N$. For any $l \in$ $\{1,2,3,4,5\}$, denote as $R_{S}^{l, t}$ any subprofile of preferences of agents in $S$, where for any $j \in S, R_{j}^{l, t}$ is such that its restriction to $\{x, y, z\}$ coincides with $R^{l}$ in the above fact. 
Let us point out that the following three results apply for any subdomain of single-dipped preferences profiles. The first lemma states a property that any subdomain of single-dipped preferences must satisfy. Lemma 2 guarantees, when considering strategy-proof rules, the existence of two individual preferences for any triple $t$ of alternatives in the range, one that is "leftist on $t$ " and one that is "rightist on $t$ ".

Lemma 3 ensures that any alternative in the interior of the range can be obtained as the outcome of a preference profile where individual preferences are either "leftist on $t$ " or "rightist on $t$ ".

Lemma 2 Let $f$ be a strategy-proof social choice function on $\mathcal{R}_{>}^{n} \subseteq \mathcal{D}_{>}$with $\# A_{f} \geq 3$. For any triple $t$ formed by $x, y, z \in A_{f}$ such that $z>y>x$, then there exist $R^{1, t}$ and $R^{5, t} \in \mathcal{R}_{>}$.

Proof. Fix a triple $t: x, y, z \in A_{f}$ such that $z>y>x$, and let $x=f\left(R_{N}^{\prime}\right)$, $y=f\left(\widetilde{R}_{N}\right)$, and $z=f\left(\bar{R}_{N}\right)$. Suppose first that there does not exist any type 1 preference on $t$. That is, for any $R_{N} \in \mathcal{R}_{>}^{n}$ only preferences of type 2, 3, 4, and 5 may coexist. Then, $N$ would manipulate $f$ at $\widetilde{R}_{N}$ via $\bar{R}_{N}$ since $z=f\left(R_{N}\right) \widetilde{P}_{i} f\left(\widetilde{R}_{N}\right)=y$ for any $i \in N$ which is a contradiction to group strategy-proofness. Thus, there exist $R_{N} \in \mathcal{R}_{>}^{n}$ such that restricted to $t$ there are type 1 preferences.

Suppose that there does not exist any type 5 preference on $t$. That is, for any $R_{N} \in \mathcal{R}_{>}^{n}$ only preferences of type $1,2,3$, and 4 may coexist. Then, $N$ would manipulate $f$ at $\widetilde{R}_{N}$ via $R_{N}^{\prime}$ since $x=f\left(R_{N}^{\prime}\right) \widetilde{P}_{i} f\left(\widetilde{R}_{N}\right)=y$ for any $i \in N$ which is a contradiction to group strategy-proofness. Thus, there exist $R_{N}^{\prime \prime} \in \mathcal{R}_{>}^{n}$ such that restricted to $t$ there are type 5 preferences. This ends the proof.

Lemma 3 Let $f$ be a strategy-proof social choice function on $\mathcal{R}_{>}^{n} \subseteq \mathcal{D}_{>}$with $\# A_{f} \geq 3$. For any triple $t: x, y, z \in A_{f}, z>y>x$, then $y=f\left(R_{S}^{1, t}, R_{N \backslash S}^{5, t}\right)$ for some $S \subseteq N$.

Proof. Let $t$ be the triple $x, y, z \in A_{f}, z>y>x$, and $y=f\left(\widetilde{R}_{N}\right)$. Define $S_{l}=\left\{i \in N: \widetilde{R}_{i, t}=R^{l, t}\right\}$ for $l \in\{1,2,3,4,5\}$. Consider the set of agents in $N \backslash\left(S_{1} \cup S_{5}\right)$ and define $\bar{S}=\left\{i \in N \backslash\left(S_{1} \cup S_{5}\right): d\left(\widetilde{R}_{i}\right) \geq y\right\}$. By strategyproofness, $f\left(R_{\bar{S}}^{1, t}, \widetilde{R}_{N \backslash \bar{S}}\right)=y$. The argument is as follows: observe first that $f\left(R_{\bar{S}}^{1, t}, \widetilde{R}_{N \backslash \bar{S}}\right) \in[y, z)$, otherwise $\bar{S}$ would manipulate $f$ at $\widetilde{R}_{N}$ via $R_{\bar{S}}^{1, t}$ and 
get an outcome strictly better than $y$. Second, $f\left(R_{\bar{S}}^{1, t}, \widetilde{R}_{N \backslash \bar{S}}\right)=y$. Otherwise, if $f\left(R_{\bar{S}}^{1, t}, \widetilde{R}_{N \backslash \bar{S}}\right) \in(y, z), \bar{S}$ would manipulate $f$ at $\left(R_{\bar{S}}^{1, t}, \widetilde{R}_{N \backslash \bar{S}}\right)$ via $\widetilde{R}_{\bar{S}}$ and get $y$, since any $j \in \bar{S}, y P_{j}^{1, t} f\left(R_{\bar{S}}^{1, t}, \widetilde{R}_{N \backslash \bar{S}}\right)$. Thus, $f\left(R_{\bar{S}}^{1, t}, \widetilde{R}_{N \backslash \bar{S}}\right)=y$.

Define now $\widehat{S}=\left\{i \in N \backslash\left(S_{1} \cup S_{5}\right): d\left(\widetilde{R}_{i}\right)<y\right\}$. By strategy-proofness, $f\left(R_{\widehat{S}}^{5, t}, R_{\bar{S}}^{1, t}, \widetilde{R}_{N \backslash(\bar{S} \cup \widehat{S})}\right)=y$. The argument is similar to the one above: first note that $f\left(R_{\widehat{S}}^{5, t}, R_{\bar{S}}^{1, t}, \widetilde{R}_{N \backslash(\bar{S} \cup \widehat{S})}\right) \in(x, y]$. Otherwise, $\widehat{S}$ would manipulate $f$ at $\left(R_{\bar{S}}^{1, t}, \widetilde{R}_{N \backslash \bar{S}}\right)$ via $R_{\widehat{S}}^{5, t}$ and get an outcome better than $y$ for any $j \in \widehat{S}$. Second, $f\left(R_{\widehat{S}}^{5, t}, R_{\bar{S}}^{1, t}, \widetilde{R}_{N \backslash(\bar{S} \cup \widehat{S})}\right)=y$. Otherwise, if $f\left(R_{\widehat{S}}^{5, t}, R_{\bar{S}}^{1, t}, \widetilde{R}_{N \backslash(\bar{S} \cup \widehat{S})}\right) \in(x, y), \widehat{S}$ would manipulate $f$ at $\left(R_{\widehat{S}}^{5, t}, R_{\bar{S}}^{1, t}, \widetilde{R}_{N \backslash(\bar{S} \cup \widehat{S})}\right)$ via $\widetilde{R}_{\widehat{S}}$ and get $y$, since any $j \in \bar{S}$, $y P_{j}^{5, t} f\left(R_{\widehat{S}}^{5, t}, R_{\bar{S}}^{1, t}, \widetilde{R}_{N \backslash(\bar{S} \cup \widehat{S})}\right)$. Thus, $f\left(R_{\widehat{S}}^{5, t}, R_{\bar{S}}^{1, t}, \widetilde{R}_{N \backslash(\bar{S} \cup \widehat{S})}\right)=y$.

The following result obtained by the two previous lemmata assures that for any triple $t: z>y>x$ where $x, z \in A_{f}$, if alternative $y$ is in the range then there can not exist simultaneously a type 2 and a type 4 individual preference over the triple $t$.

Theorem 2 Let $f$ be a non-constant strategy-proof social choice function on $\mathcal{R}_{>}^{n} \subseteq \mathcal{D}_{>}$. Let $x, z \in A_{f}$, and a triple $t: z>y>x$ for which there exists $R^{2, t}, R^{4, t} \in \mathcal{R}_{>}$. Then, $y \notin A_{f}$.

Proof. If $\# A_{f}=2$ the result trivially holds. Suppose that $\# A_{f} \geq 3$. By contradiction suppose that $y \in A_{f}$. Let $x, z \in A_{f}$, and the triple $t$ : $z>y>x$. By Lemma 2 there exist $R^{1, t}$ and $R^{5, t}$ in $\mathcal{R}_{>}$. Moreover, by Lemma 3, $y=f\left(R_{S}^{1, t}, R_{N \backslash S}^{5, t}\right)$ for some $S \subseteq N$.

By group strategy-proofness, $f\left(R_{S}^{1, t}, R_{N \backslash S}^{4, t}\right) \in(y, z] \cup\{x\}$ (otherwise, coalition $N \backslash S$ would manipulate $f$ at $\left(R_{S}^{1, t}, R_{N \backslash S}^{4, t}\right)$ via $R_{N \backslash S}^{1, t}$. By Lemma 1, $\left.f\left(R_{S}^{1, t}, R_{N \backslash S}^{1, t}\right)=x\right)$ and again by group strategy-proofness, $f\left(R_{S}^{1, t}, R_{N \backslash S}^{4, t}\right)=x$ (otherwise, coalition $N \backslash S$ would manipulate $f$ at $\left(R_{S}^{1, t}, R_{N \backslash S}^{5, t}\right)$ via $R_{N \backslash S}^{4, t}$ ).

By group strategy-proofness, $f\left(R_{S}^{2, t}, R_{N \backslash S}^{5, t}\right) \in[x, y) \cup\{z\}$ (otherwise, coalition $S$ would manipulate $f$ at $\left(R_{S}^{2, t}, R_{N \backslash S}^{5, t}\right)$ via $R_{S}^{5, t}$ and get $\left.f\left(R_{S}^{5, t}, R_{N \backslash S}^{5, t}\right)=z\right)$, and again by group strategy-proofness, $f\left(R_{S}^{2, t}, R_{N \backslash S}^{5, t}\right)=z$ (otherwise, coalition $S$ would manipulate $f$ at $\left(R_{S}^{1, t}, R_{N \backslash S}^{5, t}\right)$ via $\left.R_{S}^{2, t}\right)$.

By group strategy-proofness, $f\left(R_{S}^{2, t}, R_{N \backslash S}^{4, t}\right)=z$ (otherwise, coalition $N \backslash S$ would manipulate $f$ at $\left(R_{S}^{2, t}, R_{N \backslash S}^{4, t}\right)$ via $R_{N \backslash S}^{5, t}$ and get $\left.f\left(R_{S}^{2, t}, R_{N \backslash S}^{5, t}\right)=z\right)$, 
but then coalition $S$ would manipulate $f$ at $\left(R_{S}^{2, t}, R_{N \backslash S}^{4, t}\right)$ via $R_{S}^{1, t}$ and get $f\left(R_{S}^{1, t}, R_{N \backslash S}^{4, t}\right)=x$. Thus, we obtain a contradiction.

Note that Theorem 2 generalizes Theorem 1 above. And in fact Theorem 1 is a straightforward corollary of Theorem 2 . Observe that if $\mathcal{R}_{>}^{n}=\mathcal{D}_{>}$, for any $t$ in $A$ : $z>y>x$ there exist $R^{2, t}$ and $R^{4, t}$ in $\mathcal{R}_{>}$. Thus, recursively applying Theorem 2 we end up showing that the $\# A_{f} \leq 2$.

Observe that by Theorem 1, the following result straightforwardly holds.

Corollary 1 There is no strategy-proof and onto social choice function on the domain of all single-dipped preferences if $\# A \geq 3$.

In Barberà, Berga, and Moreno (2009b), we obtained a characterization of all strategy-proof rules with a binary range (that is, $A_{f}=\{x, y\}$ for some pair $x, y$ of alternatives in $A$ ) by means of two conditions that we define below. Let $X\left(R_{N}\right)=\left\{i \in N: x P_{i} y\right\}$ and $Y\left(R_{N}\right)=\left\{j \in N: y P_{j} x\right\}$ for each preference profile $R_{N} \in \mathcal{R}_{>}^{n}$.

Definition 7 A social choice function $f$ with a binary range is responsive if and only if for all $R_{N}, R_{N}^{\prime} \in \mathcal{R}_{>}^{n}$ such that $R_{h}=R_{h}^{\prime}$, for any $h \in N \backslash\left[X\left(R_{N}\right) \cup Y\left(R_{N}\right)\right] \cap N \backslash\left[X\left(R_{N}^{\prime}\right) \cup Y\left(R_{N}^{\prime}\right)\right]$, the following holds:

(1) If $X\left(R_{N}^{\prime}\right) \supseteq X\left(R_{N}\right), Y\left(R_{N}\right) \supseteq Y\left(R_{N}^{\prime}\right)$ (with at least one strict inequality), and $f\left(R_{N}\right)=x$, then $f\left(R_{N}^{\prime}\right)=x$; and

(2) If $Y\left(R_{N}^{\prime}\right) \supseteq Y\left(R_{N}\right), X\left(R_{N}\right) \supseteq X\left(R_{N}^{\prime}\right)$ (with at least one strict inequality), and $f\left(R_{N}\right)=y$, then $f\left(R_{N}^{\prime}\right)=y$.

Definition 8 A social choice function $f$ with a binary range is xy-based if and only if for all $R_{N}, R_{N}^{\prime} \in \mathcal{R}_{>}^{n}$ such that $X\left(R_{N}\right)=X\left(R_{N}^{\prime}\right), Y\left(R_{N}\right)=$ $Y\left(R_{N}^{\prime}\right)$, and $R_{h}=R_{h}^{\prime}, \forall h \in N \backslash\left[X\left(R_{N}\right) \cup Y\left(R_{N}\right)\right]$, then $f\left(R_{N}\right)=f\left(R_{N}^{\prime}\right)$.

These two conditions characterize strategy-proof social choice functions with a binary range.

Theorem 3 (see Barberà, Berga, and Moreno, 2009b) Let $f$ be a social choice function on $\mathcal{R}_{>}^{n} \subseteq \mathcal{R}^{n}$ with a binary range. Then, $f$ is strategy-proof if and only if $f$ is responsive and $x y$-based. 
We already know, from our previous work, that responsiveness and the $x y$-based condition are independent whatever the domain of preferences is. For the sake of completeness, in the following example we present two rules violating only one of them when we concentrate on the set of single-dipped preferences profiles.

Example 2 Let $N=\{1,2\}, A=\{x, y, z\}$ where $z>y>x$, and for any $i \in N, \mathcal{R}_{>}=\left\{R^{1}, R^{2}, R^{4}, R^{5}\right\}$ such that:

\begin{tabular}{|l|l|l|l|}
\hline$R^{1}$ & $R^{2}$ & $R^{4}$ & $R^{5}$ \\
\hline$x$ & $x$ & $z$ & $z$ \\
\hline$y$ & $z$ & $x$ & $y$ \\
\hline$z$ & $y$ & $y$ & $x$ \\
\hline
\end{tabular}

Note that $\mathcal{R}_{>}^{2}$ is the subset of all strict single-dipped profiles relative to the above defined order of alternatives. Observe that $f$ below satisfies xz-based but it violates responsiveness. Note also that $\widehat{f}$ satisfies responsiveness but it violates $x z$-based. Both are manipulable.

\begin{tabular}{|l|l|l|l|l|}
\hline$f$ & $R_{2}^{1}$ & $R_{2}^{2}$ & $R_{2}^{4}$ & $R_{2}^{5}$ \\
\hline$R_{1}^{1}$ & $z$ & $z$ & $x$ & $x$ \\
\hline$R_{1}^{2}$ & $z$ & $z$ & $x$ & $x$ \\
\hline$R_{1}^{4}$ & $x$ & $x$ & $x$ & $x$ \\
\hline$R_{1}^{5}$ & $x$ & $x$ & $x$ & $x$ \\
\hline
\end{tabular}

\begin{tabular}{|l|l|l|l|l|}
\hline$\widehat{f}$ & $R_{2}^{1}$ & $R_{2}^{2}$ & $R_{2}^{4}$ & $R_{2}^{5}$ \\
\hline$R_{1}^{1}$ & $z$ & $\bar{x}$ & $z$ & $z$ \\
\hline$R_{1}^{2}$ & $x$ & $x$ & $z$ & $z$ \\
\hline$R_{1}^{4}$ & $z$ & $z$ & $z$ & $z$ \\
\hline$R_{1}^{5}$ & $z$ & $z$ & $z$ & $z$ \\
\hline
\end{tabular}

Therefore, as a corollary of Theorem 3 and Theorem 1, we can state the following result.

Proposition 1 Let $f$ be a social choice function on $\mathcal{D}_{>}$with a binary range. Then, $f$ is strategy-proof if and only if $f$ is responsive and $x y$-based.

\section{Strategy-proofness on restricted single-dipped domains}

We now present three families of subdomains of single-dipped preferences. For two of them we describe classes of rules that are strategy-proof on these domains, and yet have ranges of size larger than two. For the third one, we show that even it is a subset of one of those two, we are back to the situation 
where the range of any strategy-proof rule on it can only contain at most two alternatives.

Our analysis is not exhaustive. We do not work on all possible subdomains of single-dipped preferences, nor do we claim that the rules we exhibit exhaust the set of all those satisfying strategy-proofness on the respective domains. Our aim is a more limited one, hopefully interesting for the reader. It is to show that there is no inherent association between the fact that preferences are single-dipped and the need for the range to be limited to size two, nor between this limitation of the range and the fact that only two alternatives can be the best for agents, under single-dipped preferences. These connections are only effective when all single-dipped preferences are in the domain of our rules.

We present our examples for the case where domains consist of preferences defined on a finite set of alternatives. With some changes and qualifications, our examples and results could also extend to the case where the set of alternatives is a continuum. However, as already remarked by Peremans and Storcken (1999), the ranges will still be finite in that case. Again, this is a substantial difference with the case of single-peakedness.

We will let the set of alternatives be the integer interval $A=[0,1,2, \ldots, k]$, $k \geq 3$. For $k \leq 2$, the subdomains we define below would give rise to noninteresting results. We denote by $[a, b]$ the set of integer numbers between $a$ and $b$ both included, while $(a, b)$ excludes both $a$ and $b$.

Definition 9 For any given set of alternatives of size $k$, and any integer $h$, $0<h<k$, the set $\mathcal{D}_{k h}$ of single-dipped preferences with an $h$ bias consists of all preferences $R$ that:

(1) are single-dipped, and

(2) either $h P s$ for all $s \in[h+1, k]$, or else

$(k-h)$ Ps for all $s \in[0, k-h-1]$.

Note first that condition (2) implies that alternatives 0 and $k$ will not be indifferent. Notice also that if $h=0$, condition (2) would have no bite and we would be back to the case of single-dipped preferences, where 0 and $k$ are not indifferent. Moreover if $h=k-1$, then the only two admissible preferences are those where 0 is the best and $k$ is the worst alternative, or its opposite.

Notice also that, fixed $k, \mathcal{D}_{k h^{\prime}} \varsubsetneqq \mathcal{D}_{k h}$ where $0<h<h^{\prime}<k$. Furthermore, observe that if $h<\frac{k}{2}$, the set on which the dips of agents with tops in 0 and 
agents with tops on $k$ can be located are overlapping, and their intersection is the segment $I^{h}=(h, k-h)$ whereas, if $h \geq \frac{k}{2}$, then the set $I^{h}$ is always empty, and the preferences of all agents in the segment $C^{h}=[k-h, h]$ are strictly increasing or strictly decreasing. These two subdomains will allow for different types of strategy-proof rules.

We now present, for each of these two domains, examples of group strategyproof rules whose range size is larger than two.

Example 3 For $k \geq 3,0<h<\frac{k}{2}$, define a rule on $\mathcal{D}_{k h}$ as follows:

Fix a range consisting of four outcomes, $r_{1}, r_{2}, r_{3}, r_{4}$ such that $r_{1}, r_{2} \leq h$ and $r_{3}, r_{4} \geq k-h$.

For a given profile $R_{N} \in\left(\mathcal{D}_{k h}\right)^{n}$, then

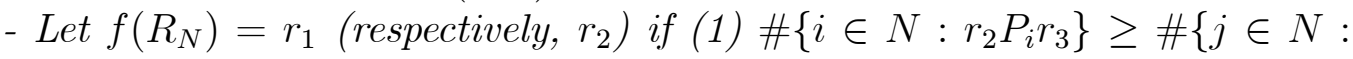
$\left.r_{3} P_{j} r_{2}\right\}$ and

(2) $\#\left\{i \in N: r_{3} P_{i} r_{2}\right.$ and $\left.r_{1} P_{i} r_{2}\right\} \geq \#\left\{j \in N: r_{3} P_{j} r_{2}\right.$ and $\left.r_{2} P_{j} r_{1}\right\}$ (respectively, $<)$.

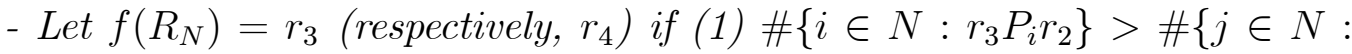
$\left.r_{2} P_{j} r_{3}\right\}$, and

(2) $\#\left\{i \in N: r_{2} P_{i} r_{3}\right.$ and $\left.r_{3} P_{i} r_{4}\right\} \geq \#\left\{j \in N: r_{2} P_{j} r_{3}\right.$ and $\left.r_{4} P_{j} r_{3}\right\}$ (respectively, <).

Informally, we could describe the rule as follows. Agents first vote by majority whether the outcome should be in $\left\{r_{1}, r_{2}\right\}$ or $\left\{r_{3}, r_{4}\right\}$, and then, those disagreeing with this majority vote again by majority to determine which of the two chosen alternatives should come out.

The rule is strategy-proof, and its range contains, by construction, four elements. The argument for (individual) strategy-proofness is as follows. Since preferences are single-dipped with a bias, all agents either prefer both $r_{1}$ and $r_{2}$ to both $r_{3}$ and $r_{4}$, or vice-versa. Hence, they will try to ensure that the outcome is any one of the two that they prefer, and no voter has a better strategy than supporting their best pair. In the second vote, agents who did not get their best alternatives pre-selected can still express their preferences between the other two, and either support the most extreme outcome or else get the less extreme one. Again, supporting their preferred alternative in this new binary vote is a dominant strategy. Group strategy-proofness is derived from Remark 1, that our domain satisfies sequential inclusion.

This rule provides an example of how, by restricting the domain of definition of our social choice functions, we may get group strategy-proof rules 
with a range larger than two. Remark that, contrary to what happened in richer domains, the rule we propose is strategy-proof but requires information beyond knowing what is the preferred alternative of each agent on the range. This is worth remarking because for many domains it is known that strategy-proof rules must only use information regarding the "tops on the range" of individual preferences. Our domains are such that this informational simplicity requirement can be skipped.

The choice of a range in this example is not capricious. The following theorem proves that, in the domain $\mathcal{D}_{k h}$ with $h<\frac{k}{2}$, this is the larger size of ranges admitting a strategy-proof rule.

Proposition 2 Let $k \geq 3,0<h<\frac{k}{2}$. There is no strategy-proof social choice function $f:\left(\mathcal{D}_{k h}\right)^{n} \rightarrow A$ with $\# A_{f}>4$.

Proof. The proof consists of two steps.

Step 1: If $\# A_{f} \geq 3$, no alternative in the interval $(h, k-h)$ belongs to the range of $f$.

Proof of Step 1: Let $y \in(h, k-h)$ and let $x, z \in A_{f}$. Observe that wherever the triple $t: x, y, z$, belongs to and for any possible order of them, there exist some preferences $R^{2, t}$ and $R^{4, t}$ in $\mathcal{D}_{k h}$.

If $z>y>x$, by Theorem $2, y \notin A_{f}$.

If $z>x>y$ (respectively, $y>z>x$ ), if we assume that $y \in A_{f}$ then by Theorem 2 we obtain that $x \notin A_{f}$ (respectively, $z \notin A_{f}$ ) which is a contradiction. This shows Step 1.

Thus $A_{f} \subseteq[0, h] \cup[k-h, k]$.

Step 2: \# $\left(A_{f} \cap[0, h]\right) \leq 2$ and $\#\left(A_{f} \cap[k-h, k]\right) \leq 2$.

Proof of Step 2: Let us show that \# $\left(A_{f} \cap[k-h, k]\right) \leq 2$ (a similar argument would follow to show that $\left.\#\left(A_{f} \cap[0, h]\right) \leq 2\right)$. Suppose that there exists $S \subseteq[k-h, k] \cap A_{f}$ such that $\# S \geq 3$. Observe that for any triple in $[k-h, k]$, there exist some preferences $R^{2, t}$ and $R^{4, t}$ in $\mathcal{D}_{k h}$. Thus, fixed any pair $x^{0}, z^{0} \in S, z^{0}>x^{0}$, for any $y \in S$ such that $z^{0}>y>x^{0}, y \notin A_{f}$ by Theorem 2. Repeatedly applying Theorem 2 for all different pairs $x^{1}, z^{1} \in S$, $x^{0} \geq x^{1}, z^{1} \geq z^{0}$ we obtain that $\# S \leq 2$ which is a contradiction and shows Step 2.

Thus $\# A_{f} \leq 4$.

Now, let us consider the following rules for the domain $\mathcal{D}_{k h}$ with $h>\frac{k}{2}$. 
Example 4 For $k \geq 3, \frac{k}{2}<h<k-1$ define a rule on $\mathcal{D}_{k h}$ as follows:

Fix four outcomes, $r_{1}, r_{2}, r_{3}, r_{4}$ such that $0 \leq r_{1}, r_{2}<k-h$ and $h<r_{3}, r_{4} \leq k$. For a given profile $R_{N} \in\left(\mathcal{D}_{k h}\right)^{n}$,

- Let $f\left(R_{N}\right)=r_{1}$ (respectively, $r_{2}$ ) if (1) $0 P_{1} k, 0 P_{2} k$, and (2) \# $\#\{\in N \backslash\{1,2\}$ : $\left.r_{1} P_{i} r_{2}\right\} \geq$ (respectively, $\left.<\right) \#\left\{j \in N \backslash\{1,2\}: r_{2} P_{j} r_{1}\right\}$.

- Let $f\left(R_{N}\right)=r_{4}$ (respectively, $\left.r_{3}\right)$ if (1) $k P_{1} 0, k P_{2} 0$ and (2) $\#\{i \in N \backslash\{1,2\}$ : $\left.r_{4} P_{i} r_{3}\right\} \geq$ (respectively, $\left.<\right) \#\left\{j \in N \backslash\{1,2\}: r_{3} P_{j} r_{4}\right\}$.

- Let $f\left(R_{N}\right)=\min \left\{h, k-h+\#\left\{i \in N \backslash\{1,2\}: k P_{i} 0\right\}\right.$ if $0 P_{1} k$ and $k P_{2} 0$ or $k P_{1} 0$ and $0 P_{2} k$.

Informally, we could describe the rule as follows. If agents 1 and 2 agree on their top then they select only two alternatives out of which the rest of agents will have to choose one. If agents 1 and 2 disagree on their top then the outcome will be an alternative within the interval $[k-h, h]$ and outcome will depend again on the preferences of the remaining agents (notice that their preferences on this interval will be either leftist or rightist). Let us briefly describe why this rule is strategy-proof. Since their preferences have an $h$ bias, if 1 and 2 both prefer 0 to $k$, then they both prefer $r_{1}$ and $r_{2}$ to all other alternatives in the range. Hence if both agree that 0 is best, it is optimal for them to declare 0 and to obtain either $r_{1}$ or $r_{2}$. Similarly if both agree that $k$ is best. And if agents 1 and 2 disagree, then it is best for both of them to avoid extreme outcomes $r_{1}, r_{2}, r_{3}$ and $r_{4}$ and to ensure through their sincere vote that the outcome lies in the range $[k-h, h]$. Given the votes of 1 and 2, the choices of the rest of the agents are either a binary election or else a vote on their best element on $[k-h, h]$ resulting in some outcome in this interval. In each of these three cases, being truthful is a dominant strategy for all them.

Let us remark that depending on $k, h$ and $n$, the range of these functions can be as large as $\min \left\{2 h-k+5,2^{n}\right\}$.

In fact, this example provides an upper bound for the size of the range of strategy-proof rules on $\mathcal{D}_{k h}$ with $h>\frac{k}{2}$, as proven in the following proposition.

Proposition 3 Let $k \geq 3$ and $\frac{k}{2}<h<k-1$. There does not exist any strategy-proof social choice function $f:\left(\mathcal{D}_{k h}\right)^{n} \rightarrow A$ such that $\# A_{f}>$ $\min \left\{2 h-k+5,2^{n}\right\}$.

Proof. Let $h \leq k-2$. We only need to show Step 1:

Step 1: $\#\left(A_{f} \cap(0, h)\right) \leq 2$ and $\#\left(A_{f} \cap(k-h, k)\right) \leq 2$. 
If these two statements in Step 1 hold, then the maximum number of alternatives in the range is four plus the maximum number of alternatives in the range that belong to the interval $[k-h, h]$. That is, \# $A_{f} \leq 2+2+(h-[k-$ $h-1])=2 h-k+5$. This would end the proof of this Proposition.

Proof of Step 1: Let us show that $\#\left(A_{f} \cap(0, h)\right) \leq 2$ (a similar argument would follow to show that $\left.\#\left(A_{f} \cap(k-h, k)\right) \leq 2\right)$. Suppose that there exists $S \subseteq(0, h) \cap A_{f}$ such that $\# S \geq 3$. Observe that for any triple in $(0, h)$, there exist some preferences $R^{2, t}$ and $R^{4, t}$ in $\mathcal{D}_{k h}$. Thus, fixed any pair $x^{0}, z^{0} \in S, z^{0}>x^{0}$, for any $y \in S$ such that $z^{0}>y>x^{0}, y \notin A_{f}$ by Theorem 2. Repeatedly applying Theorem 2 for all different pairs $x^{1}, z^{1} \in S, x^{0} \geq x^{1}$, $z^{1} \geq z^{0}$ we obtain that $\# S \leq 2$ which is a contradiction. This shows Step 1 .

Finally, let us consider a third subdomain of preferences with an $h$ bias, one that restricts the domain $\mathcal{D}_{k h}$ with $h<\frac{k}{2}$. Specifically, we will consider the set $\widehat{\mathcal{D}}_{k h}$ :

Definition 10 Let $\widehat{\mathcal{D}}_{k h}$ be the domain formed by preferences that (1) belong to $\mathcal{D}_{k h}$ with $h<\frac{k}{2}$ and

(2) $d\left(R_{i}\right) \in I^{h}=(h, k-h)$.

It turns out that, like in Theorem 1, it is now only possible to define strategy-proof rules on this subdomain of single-dipped preferences if the range is restricted to two alternatives at most, as shown by the following result.

Proposition 4 Let $k \geq 3,0<h<\frac{k}{2}$. There is no strategy-proof social choice function $f:\left(\widehat{\mathcal{D}}_{k h}\right)^{n} \rightarrow A$ with $\# A_{f}>2$.

Proof. The proof consists of two steps.

Step 1: \# $\left(A_{f} \cap[0, h]\right) \leq 1$ and $\#\left(A_{f} \cap[k-h, k]\right) \leq 1$.

Proof of Step 1: Observe that for any $R \in \widehat{\mathcal{D}}_{k h}, R$ is strictly decreasing in $[0, h]$ and strictly increasing in $[k-h, k]$. Suppose that there exist $x_{1}, x_{2} \in A_{f} \cap[0, h]$ where $x_{1}=f\left(R_{N}\right), x_{2}=f\left(\widetilde{R}_{N}\right)$. Then, $N$ would manipulate $f$ at $\widetilde{R}_{N}$ via $R_{N}$ which is the desired contradiction. A similar argument holds for $A_{f} \cap[k-h, k]$. This shows Step 1 .

Step 2: No alternative in the interval $(h, k-h)$ belongs to the range of $f$. $\overline{\text { Proof }}$ of Step 2: Let $\# A_{f} \geq 3$ and by contradiction suppose that $A_{f} \cap$ 
$(h, k-h) \neq \varnothing$. Suppose first that $A_{f} \subseteq(h, k-h)$. Then we get a contradiction to Lemma 3 since for any triple $t: x, y, z \in A_{f}$, there exist $R^{2, t}$ and $R^{4, t} \in \widehat{\mathcal{D}}_{k h}$ and thus $y \notin A_{f}$. Second, consider the case such that $A_{f} \cap([0, h] \cup[k-h, k]) \neq \varnothing$ and $A_{f} \cap(h, k-h) \neq \varnothing$. We may have two subcases: (2.1) $x \in A_{f} \cap[0, h], z \in A_{f} \cap[k-h, k]$, and $y \in A_{f} \cap(h, k-h)$, and (2.2) $x \in A_{f} \cap[0, h], y, z \in A_{f} \cap(h, k-h)$ (or else $z \in A_{f} \cap[k-h, k]$, $\left.x, y \in A_{f} \cap(h, k-h)\right)$. For both cases, consider the triple $t: x, y, z \in A_{f}$ and observe that there does not exist $R^{1, t}$ and $R^{5, t} \widehat{\mathcal{D}}_{k h}$ on the triple $t$ which contradicts Lemma 2. Thus, the only possibility is that $A_{f} \subseteq[0, h] \cup[k-h, k]$ which shows Step 2.

Combining the results in both Steps, we obtain that $\# A_{f} \leq 2$.

\section{Conclusions}

We have highlighted the fact that, in environments where preferences are single-dipped, bounds on the size of the ranges of social choice functions arise as necessary conditions for their strategy-proofness. We have shown how these bounds result from the interaction between the number of individuals and alternatives, and most importantly from the nature of the subdomains where the functions must be defined.

One important consequence of our research in this and other papers is a full characterization of the family of group strategy-proof rules on the full domain of single-dipped preferences.

The propositions on the bounds of the size of the ranges for functions defined on different subdomains also allow us to exhibit quite unexpected results. One is the very fact that such restrictions arise: this does not happen in other well studied domain restrictions admitting strategy-proof rules, like the domain of single-peaked or separable preferences (see Moulin, 1980, Barberà, Sonnenschein, and Zhou, 1991). The other is that, in a very strong sense, the relationship between the size of single-dipped subdomains and that of the ranges of strategy-proof rules defined on them is not necessarily monotonic. We have exhibited one case where it is: this is the one where we compare the three domains $\mathcal{D}_{k h^{\prime}} \varsubsetneqq \mathcal{D}_{k h} \varsubsetneqq \mathcal{D}_{>}$, fixed $k$, and being $h<\frac{k}{2}$, and $h^{\prime}>\frac{k}{2}$, which are nested, and where indeed the maximal range size compatible with strategy-proofness increases as the domains shrink. However, we have also

identified the three domains $\widehat{\mathcal{D}}_{k h} \varsubsetneqq \mathcal{D}_{k h} \varsubsetneqq \mathcal{D}_{>}$, fixed $k$ and being $h<\frac{k}{2}$ (see 
Section 4). For these three nested domains, the admissible range size goes up, as we restrict the domain from $\mathcal{D}_{>}$to $\mathcal{D}_{k h}$, and yet it then goes down if we continue restricting the domain from $\mathcal{D}_{k h}$ to $\widehat{\mathcal{D}}_{k h}$. This definitely shows that the interactions we have unearthed are non-trivial.

\section{References}

Barberà, S., D. Berga and B. Moreno (2009a): "Individual versus group strategy-proofness: when do they coincide?", Barcelona Economics Working Paper Series, Working Paper 372.

Barberà, S., D. Berga and B. Moreno (2009b): "Strategy-proof on binary ranges", mimeo.

Barberà, S., H. Sonnenschein and L. Zhou (1991). "Voting by committees". Econometrica, 59: 595-609.

Larsson, B., and L.-G. Svensson (2006): "Strategy-proof voting on the full preference domain". Mathematical Social Sciences, 52(3): 272-287.

Manjunath, V. (2009). "Efficient and Strategy-proof Social Choice When Preferences Are Single-dipped". Mimeo.

Moulin, H. (1980). "On Strategy-proofness and Single Peakedness". Public Choice, 35: 437-455.

Peremans, W. and T. Storcken (1999): "Strategy-proofness on singledipped preferences domains". Proceedings of the International Conference, Logic, Game Theory, and Social Choice: 296-313.

Sen, A.K. and P.K. Pattanaik (1969). "Necessary and Sufficient Conditions for Rational Choice under Majority Decision," Journal of Economic Theory, 1 (2): 178-202. 